\title{
First Results of the Swiss National Surgical Site Infection Surveillance Program: Who Seeks Shall Find - ERRATUM
}

In the article by Troillet, et al. ${ }^{1}$, a typographical error introduced during the production process resulted in the last sentence of the methods section incorrectly stating that "All tests were 1-tailed." The correct sentence should read "All tests were 2-tailed." The publisher apologizes to the authors and readers for this error.

\section{REFERENCE}

1. Troillet N, Aghayev E, Eisenring M-C, Widmer AF, Swissnoso. First Results of the Swiss National Surgical Site Infection Surveillance Program: Who Seeks Shall Find. Infection Control Hosp Epidemiol 2017;38:697-704. 